\title{
Medication patterns and fertility rates in a cohort of anabolic steroid users
}

\author{
Ross A. Avant, Cameron M. Charchenko, Manaf Alom, Mary E. Westerman, Francisco Maldonado, \\ Tanner Miest, Landon Trost \\ Department of Urology, Mayo Clinic, Rochester, MN, USA \\ Contributions: (I) Conception and design: RA Avant, L Trost, CM Charchenko; (II) Administrative support: L Trost, T Miest, F Maldonado; (III) \\ Provision of study materials or patients: RA Avant, CM Charchenko, M Alom, ME Westerman; (IV) Collection and assembly of data: RA Avant, M \\ Alom, ME Westerman, T Miest; (V) Data analysis and interpretation: ME Westerman, M Alom, T Miest, F Maldonado; (VI) Manuscript writing: All \\ authors; (VII) Final approval of manuscript: All authors. \\ Correspondence to: Landon Trost, MD. 200 1st St. SW, Rochester, MN 55905, USA. Email: Trost.Landon@Mayo.edu.
}

\begin{abstract}
Background: To describe self-reported medication patterns and fertility rates in a population of anabolic steroid (AS) users.

Methods: We reviewed data from an online survey of AS users and identified a sub-group who had attempted to achieve a pregnancy with their partners while using AS. The online survey consisted of questions addressing demographics, AS use, ancillary medications, and fertility outcomes.

Results: A total of 97 men (of 231 total respondents) had attempted to achieve a pregnancy while taking AS and comprise the current cohort. The majority of men were $25-44$ years old (63.9\%), married (75.5\%) and Caucasian (88.7\%). Ancillary drug use was common with only $5.2 \%$ denying drug use other than ASs. The most common reported ancillary drugs were antiestrogens $(89.7 \%)$ and sexual enhancement medications (SEMs) (68\%). The fertility rate was $92.8 \%$, with $82.4 \%$ achieving pregnancy within one year. Interestingly, only $13.5 \%$ sought fertility evaluation with treatment required in $8.3 \%$. Age at initiation of AS use, maximum dosage utilized, yearly duration of supplementation, and number of years using steroids were not associated with a prolonged duration to pregnancy or decreased rate of pregnancy.
\end{abstract}

Conclusions: Despite continued use of ASs, this cohort's self-reported fertility rates are unexpectedly high. This is presumably related to cycling of therapy and concomitant use of fertility preserving medications.

Keywords: Testosterone; sports; illegal; substance; pregnancy

Submitted Apr 22, 2018. Accepted for publication May 02, 2018.

doi: $10.21037 /$ tau.2018.05.05

View this article at: http://dx.doi.org/10.21037/tau.2018.05.05

\section{Introduction}

Anabolic steroid (AS) use is common with a $6.4 \%$ lifetime abuse rate reported for males (1). Usage may also change with age and emphasis on sporting participation, with $4 \%$ of high school students and $5 \%$ of collegiate athletes admitting to supplementation (2). Select sports have higher rates of reported use, including 1 in 10 professional football players and nearly $70 \%$ of powerlifters noting exposure (1).

Adverse effects of AS are well reported and occur in a dose-dependent fashion (3). The general effect on the male reproductive system is that of central suppression of gonadotropins. Downstream sequelae include decreased endogenous testosterone production, testicular atrophy, and impaired spermatogenesis.

Recognition of the contraceptive potential of androgens dates back as early as 1976, with multiple contemporary randomized controlled trials confirming suppression of spermatogenesis with androgen supplementation (4). Despite the known contraceptive effect of androgens, spontaneous pregnancies while using AS are often reported within online communities of users. To reduce 
hypothalamic-pituitary-gonadal (HPG) suppression, men taking AS commonly employ medical augmentation protocols that maintain low levels of spermatogenesis during a cycle of AS and augment recovery of the HPG axis and spermatogenesis following cessation of the cycle (5-8).

To date, no studies have specifically evaluated paternity rates among men taking supra-physiologic doses of AS. Given the paucity of data and increasing interest in the concept of augmentation strategies to maintain fertility while administering testosterone for cases of male infertility and hypogonadism, we sought to describe the fertility status and medication usage in a cohort of AS users.

\section{Methods}

Following institutional review board approval, nine anonymous websites were identified using Google (www. google.com) search terms "bodybuilding forum" and "AS forum" between February and June of 2015. Moderators of each website were contacted regarding the aims, confidentiality, inclusion criteria, and survey questions of the study. With their approval, a brief description of the study and anonymous link to a survey was posted on the main forum or in the AS sub-forum, if available. The survey was created using Survey Monkey (www.surveymonkey. com), a secure third-party independent data collection service. All data was gathered in an anonymous fashion and participants were not incentivized to take the survey. Informed consent was placed on the first page of the survey and agreed to upon answering 3 inclusion criteria questions. Inclusion criteria were as follows: age $18+$, male gender, current or previous use of AS. Incomplete surveys and men receiving testosterone supplementation under the guidance of a physician were excluded from analysis. Of the men completing the survey, a subset of those who had attempted to achieve a pregnancy during AS use was reviewed and comprises the current study cohort.

The questionnaire included 49 items and took on average, 5-10 minutes to complete. All questions were voluntary with the option to skip a question or end the survey at any time. No incentives were provided to complete the survey. Survey data included: demographics, participation in sports, details of testosterone and other performance enhancing drug use, medical conditions, side effects while on and off AS, fertility, and sexual function related questions.

Post cycle therapy (PCT) was defined as a washout period from AS combined with ancillary drugs (antiestrogen,
HCG) to reestablish function of the HPG axis. The term antiestrogen included either selective estrogen receptor modulators (SERM) or aromatase inhibitors (AI).

\section{Statistics}

Student's $t$-test was used to compare continuous variables. Fisher's exact test and Pearson's chi-square test were used to compare categorical variables. Statistical analysis was performed using the JMP (SAS Institute, Cary, NC, USA; www.sas.com) software package, with $\mathrm{P} \leq 0.05$ defined as significant.

\section{Results}

A total of 323 participants were anonymously polled in a self-reporting fashion. Sixty-six were excluded for not meeting inclusion criteria, 26 surveys were incomplete, and 134 had not attempted to have children. This yielded $97(30 \%)$ surveys with usable data, with all study results reported in the current manuscript pertaining to this group. Demographics of the cohort are listed in Table 1. The majority of AS users were 25-44 years old (62.7\%), married (72.3\%), Caucasian $(88.9 \%)$, well-educated $(54.6 \%$ with at least a college degree), and earning $>\$ 75,000$ per year (57.6\%). Current or previous collegiate athletes represented $21.2 \%$ of the cohort.

Common reasons for initiating testosterone included gains in muscle mass $(36.1 \%)$ and self-assessed low testosterone $(15.5 \%)$. Notably, low testosterone was subjective and interpreted by the individual participant, with one participant initiating testosterone due to a perceived low testosterone value of $520 \mathrm{ng} / \mathrm{dL}$. Interestingly, a desire to attract women $(2.1 \%)$ and peer pressure $(3.1 \%)$ were relatively uncommon reasons for supplementing AS. See Table 2 for a list of self-reported reasons for AS use. The majority of survey respondents reported AS use for greater than one year $(85.6 \%)$ and regularly used testosterone doses $>600 \mathrm{mg} /$ week (58.8\%). Continuous utilization was also high, with $57.7 \%$ reporting testosterone use for $\geq 41$ weeks per year. Dosing and AS utilization patterns are described in Table 3.

Ancillary drug use was common with only $5.2 \%$ of patients denying use of additional drugs. The most frequently reported ancillary drugs were antiestrogens and sexual enhancement medications (SEMs) $(89.7 \%$ and $68 \%$, respectively). SEMs included phosphodiesterase inhibitors and dopamine agonists (e.g., Cabergoline). PCT 
Table 1 Demographics of AS users attempting to achieve a pregnancy

\begin{tabular}{|c|c|}
\hline Variable & Users (\%), N=97 \\
\hline \multicolumn{2}{|l|}{ Age (years) } \\
\hline $18-24$ & 4.0 \\
\hline $25-34$ & 33.3 \\
\hline $35-44$ & 29.4 \\
\hline $45-54$ & 25.3 \\
\hline $55-64$ & 0.0 \\
\hline $65+$ & 8.0 \\
\hline \multicolumn{2}{|l|}{ Race } \\
\hline White/Caucasian & 88.9 \\
\hline Hispanic/Latino & 4.0 \\
\hline American Indian/Alaskan & 2.0 \\
\hline Asian & 1.0 \\
\hline Prefer not to answer & 4.0 \\
\hline \multicolumn{2}{|l|}{2014 pre-tax income $(\mathrm{k})$} \\
\hline$<25$ & 5.1 \\
\hline $25-34,999$ & 4.0 \\
\hline $35-49,999$ & 5.1 \\
\hline $50-74,999$ & 19.2 \\
\hline $75-99,999$ & 12.2 \\
\hline $100-149,999$ & 32.3 \\
\hline$>150$ & 13.1 \\
\hline Prefer not to answer & 9.1 \\
\hline \multicolumn{2}{|l|}{ Highest level of education } \\
\hline$<12$ th grade & 6.1 \\
\hline High school diploma & 11.1 \\
\hline Some college, no degree & 24.2 \\
\hline Associate/technical degree & 16.2 \\
\hline Bachelor's degree & 22.2 \\
\hline Graduate/professional degree & 16.2 \\
\hline Prefer not to answer & 4.0 \\
\hline \multicolumn{2}{|l|}{ Marital status } \\
\hline Single & 15.2 \\
\hline Married & 72.3 \\
\hline Divorced & 8.1 \\
\hline Prefer not to answer & 1.0 \\
\hline \multicolumn{2}{|l|}{ College athlete (past/present) } \\
\hline Yes & 21.2 \\
\hline No & 78.8 \\
\hline
\end{tabular}

AS, anabolic steroid.
Table 2 Reasons for initiating AS

\begin{tabular}{lc}
\hline Reasons & Users (\%), N=97 \\
\hline Muscle mass & 36.1 \\
Low testosterone & 15.5 \\
Strength & 11.3 \\
Improve sports performance & 7.2 \\
Aesthetics & 7.2 \\
Symptoms of low testosterone & 7.2 \\
Break plateau & 5.2 \\
Compete in bodybuilding/power lifting & 4.1 \\
Heal from injury & 4.1 \\
Peer pressure & 3.1 \\
Anti-aging & 3.1 \\
Attract women & 2.1 \\
\hline
\end{tabular}

AS, anabolic steroid.

was utilized by $55.2 \%$ of respondents. Additional details of ancillary drug usage are outlined in Table 4.

A total of $92.8 \%$ of study respondents who attempted to achieve a pregnancy were able to do so, with $82.4 \%$ occurring within one year of unprotected intercourse. Eighteen participants (18.8\%) reported difficulty achieving pregnancy, with $13.5 \%$ seeking advice from a fertility specialist and $8.3 \%$ requiring medications and/or surgery. Among 32 men who were actively using testosterone while attempting to achieve a pregnancy, 29 (90.6\%) were successful. When compared to those with a history of use, but not actively using testosterone (65), there was no difference in duration to conception $(\mathrm{P}=0.11)$, rate of difficulty achieving pregnancy $(\mathrm{P}=0.51)$, evaluation by a fertility specialist $(\mathrm{P}=0.46)$, or need for medications/ surgery $(\mathrm{P}=0.56)$. The total number of children also did not significantly differ between the groups $(\mathrm{P}=0.06)$. Men achieving pregnancy while on testosterone had a longer history of use $(\mathrm{P}=0.04)$, compared to the remaining cohort, but did not utilize antiestrogens $(\mathrm{P}=0.92)$ or PCT $(\mathrm{P}=0.61)$ more frequently. Age at initiation of testosterone, number of years utilizing, maximum dosage, and number of weeks per year utilized were not associated with duration required to achieve pregnancy or rate of achieving pregnancy. Furthermore, the aforementioned variables did not affect the need for fertility evaluation or treatment (all $\mathrm{P}>0.05$ ). When considering demographic variables, age, employment status, income, level of education, and marital status were 
Table 3 AS usage patterns

\begin{tabular}{|c|c|}
\hline Variable & Users (\%), $\mathrm{N}=97$ \\
\hline \multicolumn{2}{|c|}{ Age initiated anabolic steroids } \\
\hline $14-18$ & 7.3 \\
\hline $19-22$ & 18.8 \\
\hline $23+$ & 74.0 \\
\hline \multicolumn{2}{|c|}{ Duration of use (years) } \\
\hline$<1$ & 14.4 \\
\hline $1-3$ & 32.0 \\
\hline $3-5$ & 15.4 \\
\hline $5-10$ & 18.6 \\
\hline$>10$ & 19.6 \\
\hline \multicolumn{2}{|c|}{ Weeks/year on anabolic steroids } \\
\hline$<10$ & 1.0 \\
\hline 10-20 & 16.5 \\
\hline $21-30$ & 14.4 \\
\hline $31-40$ & 10.3 \\
\hline $41-50$ & 10.3 \\
\hline$>50$ & 47.4 \\
\hline \multicolumn{2}{|c|}{ Maximum dose injected/week (mg) } \\
\hline$\leq 400$ & 15.5 \\
\hline $400-599$ & 24.7 \\
\hline $600-799$ & 15.5 \\
\hline 800-999 & 24.7 \\
\hline$\geq 1,000$ & 18.6 \\
\hline No answer & 1.0 \\
\hline
\end{tabular}

AS, anabolic steroid.

also not associated with the rate of successful pregnancy. Those using antiestrogens and PCT did not have improved fertility outcomes when compared to the remaining cohort $(\mathrm{P}>0.05)$. Full details of fertility in AS users are outlined in Table 5 .

\section{Discussion}

We report, to our knowledge, the first description of self-reported paternity rates and patterns of fertility preserving medication use in the AS user population. Conception at one year in our cohort reached $82.5 \%$
Table 4 Ancillary drug use

\begin{tabular}{lc}
\hline Ancillary drug & Users (\%), N=97 \\
\hline Antiestrogens (SERM, Al) & 89.7 \\
SEMs & 68.0 \\
17-alpha alkylated hormones & 62.9 \\
Dieting agents (clenbuterol, t3) & 51.5 \\
Other as & 44.3 \\
Research peptides & 40.2 \\
Human growth hormone & 29.9 \\
\hline
\end{tabular}

SERM, selective estrogen receptor modulators; Al, aromatase inhibitors; SEMs, sexual enhancement medications.

Table 5 Fertility of AS users

\begin{tabular}{|c|c|}
\hline Variable & Users (\%), $\mathrm{N}=97$ \\
\hline \multicolumn{2}{|l|}{ Time to pregnancy (months) } \\
\hline$<3$ & 64.9 \\
\hline $3-6$ & 9.3 \\
\hline $6-12$ & 8.2 \\
\hline$>12$ & 10.3 \\
\hline Unsuccessful/still attempting & 7.2 \\
\hline \multicolumn{2}{|l|}{ Pregnancy } \\
\hline $\begin{array}{l}\text { Actively using as while attempting } \\
\text { pregnancy }\end{array}$ & 33.7 \\
\hline Difficulty achieving pregnancy & 18.8 \\
\hline \multicolumn{2}{|l|}{ Fertility evaluation } \\
\hline Fertility consultation & 13.5 \\
\hline Required medication/surgery & 8.3 \\
\hline \multicolumn{2}{|l|}{ Number of children } \\
\hline 0 & 7.2 \\
\hline 1 & 29.9 \\
\hline 2 & 28.9 \\
\hline 3 & 14.4 \\
\hline $4+$ & 10.3 \\
\hline No response & 9.3 \\
\hline
\end{tabular}

AS, anabolic steroid.

which is only slightly lower than the $90 \%$ rate reported within the general population (9). Interestingly, 29/32 $(90.6 \%)$ of those actively using testosterone and other 
AS while attempting to conceive, were successful while using ancillary medications. This subset did not report longer durations to achieve conception or increased difficulty achieving pregnancy. These numbers are further supported by modest rates of fertility evaluation $(13.5 \%)$ and subsequent treatment (8.3\%).

Surprisingly, statistical analysis failed to demonstrate any significant associations between successful pregnancy and testosterone frequency of use, dose, or specific agents used to mitigate side effects of therapy such as antiestrogens. The only significant association identified was a longer duration of testosterone use among men who achieved a pregnancy while on testosterone compared to those that were between cycles of AS. This likely reflects the finding that older men had longer durations of testosterone use overall and were more likely to attempt to achieve a pregnancy during that time period as compared to their younger counterparts.

The HPG axis is variably suppressed following administration of exogenous testosterone $(10,11)$. A World Health Organization study performed in 1990 included 271 healthy men receiving $200 \mathrm{mg}$ of testosterone enanthate (TE) weekly over a 12 months' period. Among the participants, only 157 (65\%) became azoospermic at 6 months. Eleven participants also experienced an escape phenomenon with restoration of sperm in the ejaculate despite supra-physiologic testosterone levels. Following TE discontinuation, mean time to recovery of sperm counts $>20 \times 10^{6}$ was 3.7 months with all subjects reaching baseline sperm counts at a mean 6.7 months (6.2-8.7) (11). Recovery of the HPG axis appears to be reliable and predictable across a large population. A review of 30 clinical trials, including over 1,500 men, showed $100 \%$ recovery to nonoligospermic levels by 2 years across varying contraceptive protocols (12). Despite decreases in sperm count, TE does not appear to affect sperm function, morphology, or motility in men with normal semen parameters (13).

A small retrospective series suggests a dose independent and incomplete suppression of spermatogenesis with AS (14). Knuth and colleagues retrospectively examined 41 bodybuilders' semen analyses along with self-reported steroid administration logs. Despite steroid doses up to 40 times higher than clinical application, only 24 displayed subnormal sperm counts. Those without exposure in the previous 4 months were normospermic (15). Other case reports support recovery of normal sperm levels within 1 year of abstinence despite previously excessive doses of AS $(16,17)$.

Based on these prior reports of suppression of the
HPG axis with testosterone, one would expect lower rates of pregnancy in AS users. The relatively high rate of preservation is likely secondary to the common practice of AS users to include adjunctive therapies along with testosterone or to include washout periods between intervals of AS use (cycling). Often, the primary objective is not maintenance of fertility, but to prevent adverse effects of AS withdrawal including muscle loss, loss of libido, erectile dysfunction, and impaired mood (18). Commonly recommended drugs by online communities include human chorionic gonadotropin (HCG), SERMs, and AIs. When implemented following a cycle of AS, the steroid community refers to this practice as PCT (5). Notably, HCG is also recommended during an AS cycle to maintain testicular volume.

There is currently very limited data available on the impact of combining testosterone and secondary therapies on fertility outcomes. Karila and colleagues reported on a series of 18 healthy male power athletes utilizing massive doses of AS concomitant with HCG (6). Semen analysis was checked at the end of the cycle as well as 1.5 and 6 months later. Upon completion of the cycle, mean sperm counts were $(33 \pm 49) \times 10^{6} / \mathrm{mL}$, and only one participant was azoospermic. By six months all had recovered normal sperm counts and semen parameters. HCG administration maintained spermatogenesis, likely via maintenance of intratesticular testosterone levels, despite massive doses of exogenous hormones. All participants continued to abuse AS and 10 (55\%) had one or more children within six years of the study (6). However, no data were obtained on the percentage of men attempting to achieve a pregnancy during or following the study. Maintenance of spermatogenesis and intratesticular testosterone levels has also been reported with HCG and AIs in the presence of exogenous testosterone administration in the hormone replacement therapy population $(7,19,20)$. These studies suggest a possible mechanism for the preserved fertility rates among our cohort of men.

Our study has several notable limitations including the non-validated survey based format with associated sampling and recall bias. Data are also dependent upon the accuracy of participant reports and may be confounded by participants wishing to demonstrate that their practices are more benign than might otherwise be expected. The survey was administered via the internet which may select for a more educated, affluent, and/or motivated population. Despite these limitations, the current study provides demographics, patterns of utilization, and fertility outcomes 
from a large sampling of AS users who have pursued pregnancy.

\section{Conclusions}

Fertility rates are maintained in a population of AS users and are relatively equivalent to age-matched historical controls. This surprising finding may be related to the common use of washout periods, adjunctive medications (SERMs, AIs), and incomplete suppression of the HPG axis despite large doses of AS. The finding of maintained fertility is further validated by modest rates of infertility evaluations and/or treatment which also parallel that seen in the general population.

\section{Acknowledgements}

None.

\section{Footnote}

Conflicts of Interest: The authors have no conflicts of interest to declare.

Ethical Statement: The study was approved by institutional review board of Mayo Clinic Institutional Review Board (No. 14-009272).

\section{References}

1. Reardon CL, Creado S. Drug abuse in athletes. Subst Abuse Rehabil 2014;5:95-105.

2. Rouge-Pont F, Mayo W, Marinelli M, et al. The neurosteroid allopregnanolone increases dopamine release and dopaminergic response to morphine in the rat nucleus accumbens. Eur J Neurosci 2002;16:169-73.

3. Maravelias C, Dona A, Stefanidou M, et al. Adverse effects of anabolic steroids in athletes. A constant threat. Toxicol Lett 2005;158:167-75.

4. Grimes DA, Lopez LM, Gallo MF, et al. Steroid hormones for contraception in men. Cochrane Database Syst Rev 2012:Cd004316.

5. Karavolos S, Reynolds M, Panagiotopoulou N, et al. Male central hypogonadism secondary to exogenous androgens: a review of the drugs and protocols highlighted by the online community of users for prevention and/ or mitigation of adverse effects. Clin Endocrinol (Oxf) 2015;82:624-32.
6. Karila T, Hovatta O, Seppala T. Concomitant abuse of anabolic androgenic steroids and human chorionic gonadotrophin impairs spermatogenesis in power athletes. Int J Sports Med 2004;25:257-63.

7. Hsieh TC, Pastuszak AW, Hwang K, et al. Concomitant intramuscular human chorionic gonadotropin preserves spermatogenesis in men undergoing testosterone replacement therapy. J Urol 2013;189:647-50.

8. Menon DK. Successful treatment of anabolic steroidinduced azoospermia with human chorionic gonadotropin and human menopausal gonadotropin. Fertil Steril 2003;79 Suppl 3:1659-61.

9. Gnoth C, Godehardt E, Frank-Herrmann P, et al. Definition and prevalence of subfertility and infertility. Hum Reprod 2005;20:1144-7.

10. Kamischke A, Ploger D, Venherm S, et al. Intramuscular testosterone undecanoate with or without oral levonorgestrel: a randomized placebo-controlled feasibility study for male contraception. Clin Endocrinol (Oxf) 2000;53:43-52.

11. Contraceptive efficacy of testosterone-induced azoospermia in normal men. World Health Organization Task Force on methods for the regulation of male fertility. Lancet 1990;336:955-9.

12. Liu PY, Swerdloff RS, Christenson PD, et al. Rate, extent, and modifiers of spermatogenic recovery after hormonal male contraception: an integrated analysis. Lancet 2006;367:1412-20.

13. Wang C, Leung A, Superlano L, et al. Oligozoospermia induced by exogenous testosterone is associated with normal functioning residual spermatozoa. Fertil Steril 1997;68:149-53.

14. Torres-Calleja J, González-Unzaga M, DeCelis-Carrillo $\mathrm{R}$, et al. Effect of androgenic anabolic steroids on sperm quality and serum hormone levels in adult male bodybuilders. Life Sci 2001;68:1769-74.

15. Knuth UA, Maniera H, Nieschlag E. Anabolic steroids and semen parameters in bodybuilders. Fertil Steril 1989;52:1041-7.

16. Boyadjiev NP, Georgieva KN, Massaldjieva RI, et al. Reversible hypogonadism and azoospermia as a result of anabolic-androgenic steroid use in a bodybuilder with personality disorder. A case report. J Sports Med Phys Fitness 2000;40:271-4.

17. Gazvani MR, Buckett W, Luckas MJ, et al. Conservative management of azoospermia following steroid abuse. Hum Reprod 1997;12:1706-8.

18. de Souza GL, Hallak J. Anabolic steroids and 
male infertility: a comprehensive review. BJU Int 2011;108:1860-5.

19. Mehta A, Bolyakov A, Roosma J, et al. Successful testicular sperm retrieval in adolescents with Klinefelter syndrome treated with at least 1 year of topical testosterone and aromatase inhibitor. Fertil Steril 2013;100:970-4.

Cite this article as: Avant RA, Charchenko CM, Alom M, Westerman ME, Maldonado F, Miest T, Trost L. Medication patterns and fertility rates in a cohort of anabolic steroid users. Transl Androl Urol 2018;7(Suppl 2):S198-S204. doi: 10.21037/ tau.2018.05.05
20. Coviello AD, Matsumoto AM, Bremner WJ, et al.

Low-dose human chorionic gonadotropin maintains

intratesticular testosterone in normal men with

testosterone-induced gonadotropin suppression. J Clin

Endocrinol Metab 2005;90:2595-602. 